\title{
CNO ABUNDANCES OF STARS UNDERGOING FIRST DREDGE UP MIXING
}

\author{
CHRISTOPHER SNEDEN \\ Department of Astronomy and McDonald Observatory \\ The University of Texas \\ Austin Texas 78712 \\ USA
}

\begin{abstract}
The abundances of $\mathrm{C}, \mathrm{N}, \mathrm{O}$ and the $\mathrm{C}$ and $\mathrm{O}$ isotopic ratios of stars undergoing the first dredge up phase of convective mixing are summarized in this paper. It is shown that rough agreement between predictions and observations of the surface abundances may be achieved for young Pop I giants, but that the observations deviate increasingly from the predictions in old disk field giants and especially in Pop II field and globular cluster giants. Recent investigations containing new information on the correlations among these elements in globular cluster giants are discussed.
\end{abstract}

\section{Introduction}

The first dredge up mixing episode in a star occurs during the evolution from the main sequence through the subgiant branch to the tip of the giant branch. The surface convection zone deepens as the star expands and eventually comes in contact with those interior portions that have undergone hydrogen fusion reactions via the $\mathrm{p}-\mathrm{p}, \mathrm{CN}$ and possibly even the $\mathrm{ON}$ cycles. Through this mixing of the interior and envelope layers the surface composition of the CNO group is expected to be altered. We have understood the qualitative effects of this mixing for a couple of decades. The ${ }^{4} \mathrm{He}$ content will rise, but of course this is virtually unobservable spectroscopically in giant stars. More relevantly, the surface ${ }^{12} \mathrm{C}$ gradually will be depleted in favor of the $\mathrm{CN}$-cycle products ${ }^{13} \mathrm{C}$ and ${ }^{14} \mathrm{~N}$. If the convective envelope can penetrate farther into hotter interior layers then the effects of the ON cycle may be seen at the surface: decreased ${ }^{16} \mathrm{O}$ and further increase in ${ }^{14} \mathrm{~N}$. The surface abundance of ${ }^{17} \mathrm{O}$ can be enhanced, with the magnitude of the effect probably dependent on the mass of the giant, and ${ }^{18} \mathrm{O}$ is left virtually unchanged by the first dredge up. Perhaps at one time we thought that we understood the first dredge up quantitatively as well, but observational data from recent years certainly have brought about a more complex picture of this event.

Most studies of the CNO abundances in evolved stars have begun necessarily with the assumption that in the main sequence progenitors of these giants the initial abundance ratios among the CNO elements were solar. For our discussion we adopt the solar abundances and isotopic ratios recommended by Anders and Grevesse (1989; see also the extensive discussion of the derivation of reliable solar abundances given by Lambert 1978): $\mathrm{C} / \mathrm{N}=3.2 \pm 0.7, \mathrm{O} / \mathrm{C}=2.3 \pm$ $0.5,{ }^{12} \mathrm{C} /{ }^{13} \mathrm{C} \approx 90,{ }^{16} \mathrm{O} /{ }^{17} \mathrm{O} \approx 2600$, and ${ }^{16} \mathrm{O}^{18} \mathrm{O} \approx 500$.

Greene (1969) published a pioneering study of the CNO abundances in 4 bright field giant stars which showed that the $\mathrm{C} / \mathrm{N}$ ratios of these stars are sensibly different than the sun's, and that the $\mathrm{O}$ 
abundance is essentially solar. The abundances deduced by Greene matched roughly the predictions by Iben $(1967 \mathrm{a}, \mathrm{b})$ of the surface abundances for first ascent giants. However, some early studies provided clues that modifications to the standard picture of the CNO elements in giants would become necessary. For example, for the old disk giant $\alpha$ Boo, Lambert and Dearborn (1972) and Day et al. (1973) demonstrated that the carbon isotope ratio is anomalously low: ${ }^{12} \mathrm{C} /{ }^{13} \mathrm{C} \approx 7.2$. Also for $\alpha$ Boo, Conti et al. (1967) presented qualitative arguments to suggest a high $O$ abundance: $[\mathrm{O} / \mathrm{Fe}] \approx+0.3$ (we employ the usual spectroscopic notation that $[\mathrm{X}]$ $\left.\equiv \log _{10}(X)_{\text {star }}-\log _{10}(X)_{\text {sun }}\right)$. Finally, Sneden's $(1973,1974)$ values of $C / N \approx 0.2$ in some Pop II very metal-poor field giants were significantly lower than Iben's predictions.

Many studies of CNO elements in large numbers of evolved stars of all populations have been published in recent years, and in this review we outline the major trends seen in these studies and attempt to show where they confirm first dredge up theory and where more observational and theoretical work is needed to clear up some glaring discrepancies that now are obvious in the literature. Our review will be limited to stars on the first ascent of the giant branch and those in the core helium burning red giant clump (or the red horizontal branch for metal-poor giants). Other papers at this conference consider the further mixing episodes (usually called the second and third dredge up phases). We will suggest here, following authors of many recent papers, that the preponderance of data demands the introduction of a new mixing event to occur between completion of the classical first dredge up (on the lower giant branch) and the onset of the helium core flash at the red giant tip.

\section{Carbon and Oxygen Isotopic Ratios}

The ready association of $\mathrm{C}$ into molecules and the relatively large abundance fraction of ${ }^{13} \mathrm{C}$ in evolved stars leads to relatively easy detection of such isotopic species as ${ }^{13} \mathrm{CO},{ }^{13} \mathrm{CN}$, and ${ }^{13} \mathrm{CH}$ in spectra of giant stars. Moreover, the ${ }^{12} \mathrm{C} /{ }^{13} \mathrm{C}$ isotopic ratios deduced from these species are among the easiest abundance indices to derive as well as the most reliable. The molecules formed with ${ }^{12} \mathrm{C}$ and with ${ }^{13} \mathrm{C}$ are virtually identical and thus uncertainties in the atmosphere parameters $\mathrm{T}_{\text {eff, }} \log \mathrm{g}$, and $[\mathrm{Fe} / \mathrm{H}]$ largely cancel in the formation of an isotopic ratio. Only errors in the microturbulent velocities $\xi_{\text {turb }}$ can produce substantial uncertainties in ${ }^{12} \mathrm{C} /{ }^{13} \mathrm{C}$, as the transitions formed from the molecules with ${ }^{12} \mathrm{C}$ often are saturated when those with ${ }^{13} \mathrm{C}$ may be detected.

Carbon isotope ratios have been determined now for giant stars with $+0.4 \geq[\mathrm{Fe} / \mathrm{H}] \geq-2.7$, and in Figure 1 we have gathered the ${ }^{12} \mathrm{C} /{ }^{13} \mathrm{C}$ results from some of the major surveys of $\mathrm{G}-\mathrm{K}$ giants of all stellar populations. First consider the domain of $[\mathrm{Fe} / \mathrm{H}] \geq-0.7$. The points labeled "young disk" refer to those for Pop I stars from a series of papers by Lambert and colleagues (Dearborn et al. 1975; Tomkin et al. 1975, 1976). Obvious supergiants and subgiants from these studies have been eliminated from consideration here. The $[\mathrm{Fe} / \mathrm{H}]$ assignments have come from the catalog of Cayrel de Strobel et al. (1985); for the few stars with undetermined metallicities we assumed $[\mathrm{Fe} / \mathrm{H}]=0$. The points for old disk giants have been taken from Cottrell and Sneden (1985), and those for CN weak stars come from a new study by Luck (1990). The reader should be cautioned here that the names of these stellar populations are meant to characterize the majority of the samples in the cited investigations, and should not be taken too literally. Counter examples include, $\alpha$ Boo in the young disk sample (its abundance characteristics qualify it for the old disk population), and $v^{2}$ Cnc among the old disk stars (it is quite metal-rich). The Luck CN weak sample is a heterogeneous mix of different types of giants, and we have eliminated some obviously pathological cases such as HR 6766, a so-called weak G-band star whose CNOLi abundances 
(e.g. Sneden et al. 1978) signal a very anomalous evolutionary history. It is also important to note that while all studies quoted here have derived ${ }^{12} \mathrm{C} /{ }^{13} \mathrm{C}$ ratios from the $\mathrm{CN}$ red system lines near $8000 \AA$, the spectral resolutions employed for the young disk samples were much higher than those used for the $\mathrm{CN}$ weak and old disk stars; larger uncertainties in the isotope ratios should be assessed for these stars.

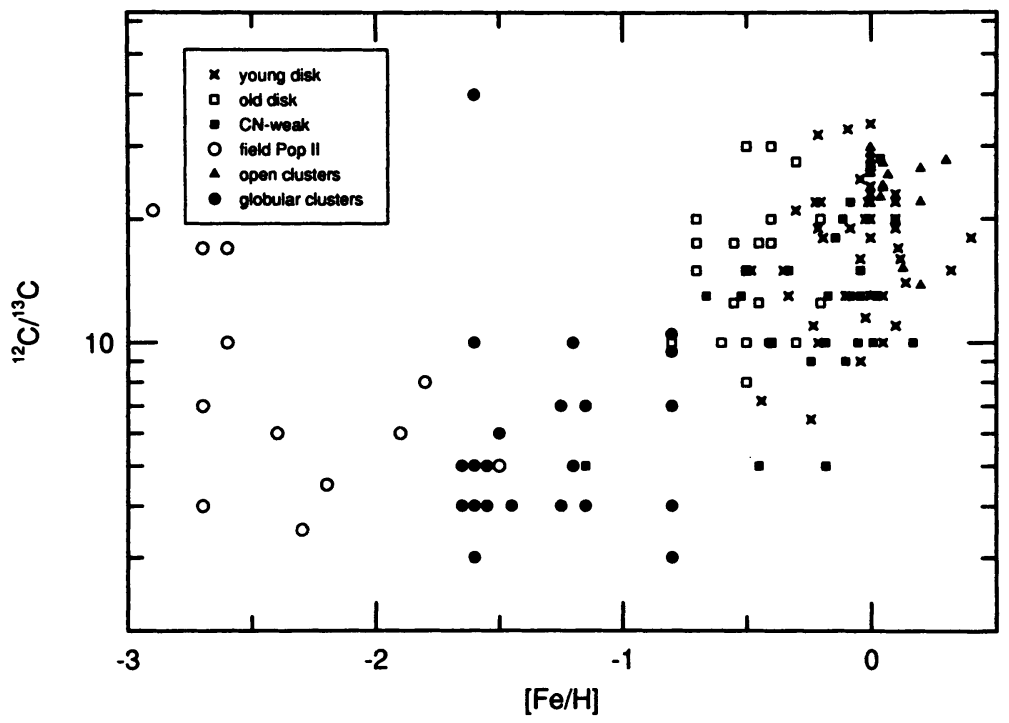

Figure 1. Carbon isotope ratios for giants as functions of their metallicities. For this figure an attempt has been made to include only ordinary giants, excluding subgiants, supergiants, and stars with very anomalous abundances.

Recently, Gilroy (1989) has completed a survey of carbon isotope ratios in giants of 20 open clusters, and average ${ }^{12} \mathrm{C} /{ }^{13} \mathrm{C}$ values for each cluster of her sample also have been plotted among the metal-rich points of Figure 1. Our Figure 1 admittedly hides the most important result of her study, namely that there is a correlation between isotope ratio and mass of these giants. For $M \geq$ $2.2 \mathrm{M}_{\odot},{ }^{12} \mathrm{C} /{ }^{13} \mathrm{C}=26 \pm 3$, irrespective of mass and in agreement with standard first dredge up theory, but for $\mathrm{M}<2.2 \mathrm{M}_{\odot}$ there is a steep decline in ${ }^{12} \mathrm{C} /{ }^{13} \mathrm{C}$ with decreasing mass. These results quantify for the first time some previous suggestions (e.g. Lambert and Ries 1981) that theoretical predictions are inadequate to account for the CNO abundances of lower mass stars. Gilroy's (1989) Figure 9 not only shows the observed trend of isotope ratios with stellar masses but emphasizes the clash with theory, as the isotopic ratios computed by Dearborn et al. (1976) and Dearborn (1990) predict increases in ${ }^{12} \mathrm{C} /{ }^{13} \mathrm{C}$ with decreasing mass. There is no obvious correlation of these ratios with cluster metallicities, but Boesgaard (1989) has shown that metallicities of open clusters have a small spread and are not obvious functions of cluster ages.

We expect a loose correlation between masses and metallicities of giants, and thus the carbon isotope ratios ought to be smaller in old disk stars, whose $[\mathrm{Fe} / \mathrm{H}]$ values and masses both are smaller than those of the young disk stars. The data of Figure 1 confirm this expectation, but only when mean values of ${ }^{12} \mathrm{C} /{ }^{13} \mathrm{C}$ for each group are computed. This is an indication of the largest problem facing interpretation of isotope ratios: the stars of the metal-rich domain of Figure 1 are 
from a mixture of evolutionary states. Direct comparisons of the isotope ratios for many of the stars prove to be nearly impossible. Evolutionary confusion is especially acute for stars with $B-V$ $\approx 1$ and $M_{\mathrm{V}} \approx 1$. These stars may be either partially mixed first ascent giants or core helium burning "clump" giants (see e.g. Brown et al. 1989 for a discussion of stars in this region of the HR diagram). This point will be addressed again (for old disk stars) in the next section.

Available ${ }^{12} \mathrm{C} /{ }^{13} \mathrm{C}$ results for metal-poor $([\mathrm{Fe} / \mathrm{H}]<-0.7)$ halo stars presented in Figure 1 show striking contrast to the ratios of metal-rich stars. Sneden et al. (1986) first demonstrated from analysis of high resolution spectra of $\mathrm{CH}$ features that 9 field Pop II giants with $M_{\mathrm{V}}<+1$ all possess ${ }^{12} \mathrm{C} /{ }^{13} \mathrm{C} \leq 10$. This result was confirmed and strengthened considerably by the work of Smith and Suntzeff (1989). They detected strong ${ }^{13} \mathrm{CO}$ bandheads in stars near the giant branch tips of 2 globular clusters and derived very low isotope ratios for most of these stars. These data sets have been supplemented further by recent work on globular cluster giants by Brown and Wallerstein (1989) and by Bell et al. (1990). In each of these studies the necessity of invoking internal mixing to achieve the very low ${ }^{12} \mathrm{C} /{ }^{13} \mathrm{C}$ ratios is stressed. Supporting evidence for this claim comes from the observation that the carbon isotope ratios are much higher $(\geq 30$, and probably higher in some cases) in low luminosity metal-poor giants and subgiants (Sneden et al. 1986) and in the metal-poor dwarf Gmb 1830 (Lambert and Sneden 1977, Sneden et al. 1986).

Although these isotopic ratios point to very efficient internal mixing in metal-poor giants, agreement between theory and observation may be achieved only through invocation of an extra mixing event beyond that predicted by standard first dredge up theory. In most papers some discussion is given to the idea of so-called meridional circulation (first introduced theoretically by Sweigart and Mengel 1979), but in reality the identification of the source of extra mixing is not possible at this time. Sneden et al. (1986) performed numerical tests to demonstrate the effects of artificially induced mixing, and the reader is referred to their paper for detailed speculations on the circumstances that lead to very low ${ }^{12} \mathrm{C} /{ }^{13} \mathrm{C}$ values. The necessity of extreme efficiency in this mixing process will be demonstrated in a forthcoming paper by Smith and Suntzeff (1990, discussed in more detail in the next section), who show that essentially all evolved stars in M4 and M22 have isotope ratios near the $\mathrm{CN}-$-cycle equilibrium value of ${ }^{12} \mathrm{C} /{ }^{13} \mathrm{C} \approx 4$.

Less will be said here on oxygen isotope ratios. In a series of papers Lambert and colleagues have explored the ${ }^{16} \mathrm{O} /{ }^{17} \mathrm{O}$ and ${ }^{16} \mathrm{O} /{ }^{18} \mathrm{O}$ ratios in a number of giants in different evolutionary states. Smith (1990) has assembled data to suggest a striking regularity in the values of ${ }^{16} \mathrm{O} /{ }^{17} \mathrm{O}$ found for ordinary giants. His Figure 2 first shows that an anticorrelation exists between the observed ${ }^{16} \mathrm{O} /{ }^{17} \mathrm{O}$ ratios (Harris et al. 1988) and ${ }^{12} \mathrm{C} /{ }^{13} \mathrm{C}$ ratios (from papers cited above). $\mathrm{He}$ then assumes that Gilroy's (1989) positive correlation between observed ${ }^{12} \mathrm{C} /{ }^{13} \mathrm{C}$ ratios and masses of open cluster giants applies equally to the field giants. This allows a mapping of the ${ }^{16} \mathrm{O} /{ }^{17} \mathrm{O}$ versus ${ }^{12} \mathrm{C} /{ }^{13} \mathrm{C}$ plot to a ${ }^{16} \mathrm{O} /{ }^{17} \mathrm{O}$ versus mass plot. This trend of a ${ }^{16} \mathrm{O} /{ }^{17} \mathrm{O}$ ratio decrease with increasing stellar mass is in excellent qualitative and reasonable quantitative agreement with theoretical predictions (Dearborn 1990, and references in Harris et al. 1988). This is a happy result, but we must caution the reader that the theoretical computations that seem to work so well for ${ }^{16} \mathrm{O} /{ }^{17} \mathrm{O}$ do not predict the observed decline of ${ }^{12} \mathrm{C} /{ }^{13} \mathrm{C}$ with mass; only an assumption of the universal applicability of Gilroy's (1989) results allows the ${ }^{16} \mathrm{O} /{ }^{17} \mathrm{O}$ trend with mass to appear so clearly.

\section{Carbon and Nitrogen Abundances}

We consider these 2 elements together since the destruction of $\mathbf{C}$ enhances $\mathbf{N}$ and the spectroscopic 
analyses of these elements often are linked. In histograms plotted in Figure 2 we summarize the C/N ratios for ordinary giants of the same stellar populations discussed in Section 2. The data for the young disk giants are taken from Lambert and Ries (1981), and those for the old disk giants are from Cottrell and Sneden (1985). Luck's (1990) results for CN weak giants are shown in a separate panel. Space here does not permit an extensive discussion of the many papers published on $\mathrm{C}$ and $\mathrm{N}$ abundances in globular cluster stars, so in Figure 2 we have chosen to display one typical example, M13, from the study of Suntzeff (1981). To be conservative about the globular cluster $\mathrm{C} / \mathrm{N}$ ratios we have chosen to apply a +0.4 dex correction tentatively suggested by Langer et al. (1985). However, even with this upward revision, the C/N ratios of M13 giants (and those of giants in other globular clusters as well) are substantially lower than giants of more metal-rich populations.

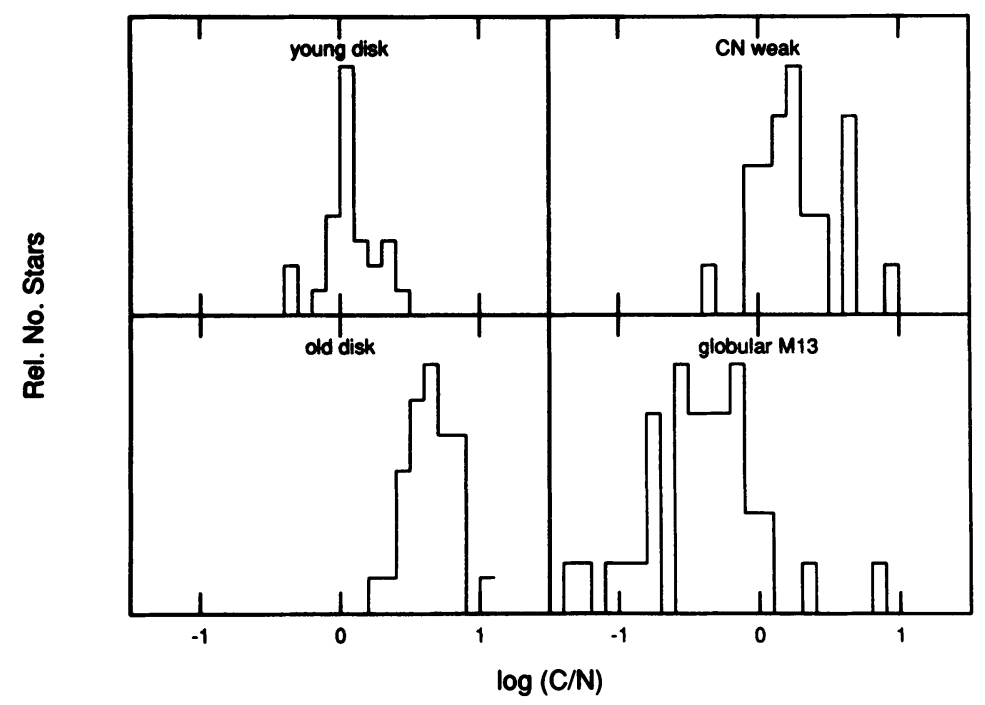

Figure 2. Distributions of $\mathrm{C} / \mathrm{N}$ ratios among ordinary giants of different stellar populations. The cluster M13 has been chosen for illustration, and is not meant to represent completely the variety of possible $\mathrm{C} / \mathrm{N}$ ratios that have been observed in globular clusters.

As with ${ }^{12} \mathrm{C} /{ }^{13} \mathrm{C}$, the $\mathrm{C} / \mathrm{N}$ ratios of young disk stars may be fit fairly well into the standard first dredge up scheme. The mean observed value is $\langle\log (\mathrm{C} / \mathrm{N})\rangle \approx 0.0$ using the Lambert and Ries data only. Kjærgaard et al. (1982) derived values of this ratio about 0.2 dex larger for giants in common with Lambert and Ries, and demonstrated that the difference could be attributed mostly to different choices in model atmosphere parameters. These results are in good accord with the predictions of Iben (1967a,b) and with those of Dearborn et al. (1976). Substantial conflicts begin to occur when considering the old disk stars. Lambert and Ries (1981) developed arguments that slow circulation currents in lower mass stars could, during their long main sequence lifetimes, expose large parts of their envelopes to interior regions where temperatures would be high enough to process ${ }^{12} \mathrm{C}$ into ${ }^{13} \mathrm{C}$ but not high enough to complete the conversion into ${ }^{14} \mathrm{~N}$. The sample of Cottrell and Sneden, specifically chosen to isolate such low mass giants, seems to confirm this notion: the old disk stars, while having lower average carbon isotope ratios than young disk stars, 
have $\langle\log (\mathrm{C} / \mathrm{N})\rangle \approx+0.6$ (see Figure 2 ), essentially the unprocessed solar ratio. The reader should note that, just as in the discussion of carbon isotope ratios, some of the spreads in C/N ratios exhibited in the 3 different field star samples are due to heterogeneity of the samples themselves: a few of the Lambert-Ries stars probably should be included in the old disk category, and the reverse is true for the Cottrell-Sneden stars. This problem is especially acute for the stars of Luck's survey, which was based only on the apparent weakness of the $\mathrm{CN}$ bands, and contains an admixture of stars from both young and old disk populations.

More importantly however we must return to the earlier caution about the dangers of comparing giants in different stages of evolution. Some recent work on the old open cluster M67 can illuminate this problem. Brown (1987) demonstrated from low resolution spectra of $\mathrm{CH}$ and $\mathrm{CN}$ that an abrupt drop from the subgiant branch value of $\log (\mathrm{C} / \mathrm{N}) \approx+0.5$ to the giant branch value of $\approx+0.0$ (converting his differential values relative to $\beta$ Gem to approximate absolute abundances) occurs at an absolute magnitude $+3.5 \geq M_{\mathrm{V}} \geq+2.8$, but his data were too few to discern any additional alterations in the $\mathrm{C} / \mathrm{N}$ ratio with further evolution. However, Gilroy and Brown (1990) do find a pronounced trend of carbon isotope ratios with $\mathrm{HR}$ diagram positions in this cluster: for 2 subgiants with $M_{\mathrm{V}} \approx+3,{ }^{12} \mathrm{C} /{ }^{13} \mathrm{C} \geq 20$ and $\geq 40$; for 4 first ascent giants with $+2.7 \geq M_{\mathrm{V}} \geq$ $+0.8,\left\langle{ }^{12} \mathrm{C} /{ }^{13} \mathrm{C}\right\rangle=22.5 \pm 1$; for 2 giant branch tip stars with $M_{\mathrm{V}} \approx+0.2,\left\langle{ }^{12} \mathrm{C} /{ }^{13} \mathrm{C}\right\rangle=14.5 \pm$ 0.5 ; and for 6 helium burning clump stars, with $\left.M_{\mathrm{V}} \geq+1.0<{ }^{12} \mathrm{C} /{ }^{13} \mathrm{C}\right\rangle=12.5 \pm 1$. The obvious implication of these changing ratios is the existence of an extra mixing event shortly before or during the helium core flash in these giants.

This work inspires a reexamination of the carbon isotope ratios in field old disk giants. A look at the Cottrell and Sneden (1985) paper should suggest to the reader that their data were adequate for determinations of $\mathrm{C} / \mathrm{N}$ in these stars but quite marginal for ${ }^{12} \mathrm{C} /{ }^{13} \mathrm{C}$ ratios. Indeed, one notices that isotope ratios are not quoted in their paper for many of the lower luminosity giants. We

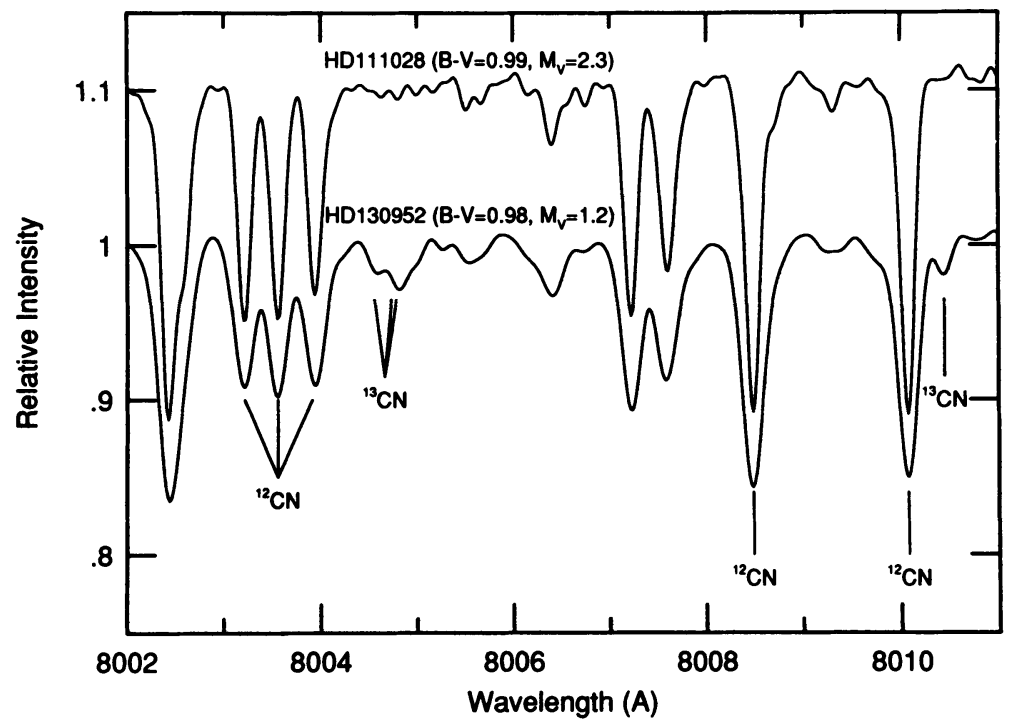

Figure 3. The spectra of 2 old disk giants from the Cottrell and Sneden (1985) sample. These stars have been chosen to be nearly identical in effective temperatures and metallicities, but they differ by about 1 magnitude in $M_{\mathrm{V}}$. 
(Sneden and Pilachowski, in preparation) have been gathering new very high resolution spectra of $\mathrm{CN}$ and $\mathrm{Li}$ features to describe better the onset of the first dredge up in these stars. The full analyses have not been done yet, but the variations in ${ }^{12} \mathrm{C} /{ }^{13} \mathrm{C}$ may be seen easily in the raw data. In Figure 3 we show the spectra of 2 stars with essentially the same temperatures but separated in luminosity by about 1 magnitude. Clearly the higher luminosity star has a higher surface carbon isotope ratio than does the lower luminosity star.

For the present we have chosen to describe the ${ }^{12} \mathrm{C} /{ }^{13} \mathrm{C}$ ratio simply by an equivalent width ratio of the line at $8010.08 \AA\left({ }^{12} \mathrm{CN}\right)$ to the triplet of lines at $8004.6 \AA\left({ }^{13} \mathrm{CN}\right)$. The ratios for the Cottrell and Sneden stars stars observed to date have been divided into groups with large $(>10)$ values of E.W.8010 $/ \mathrm{E}$.W.8004 and those with small values of this ratio. These stellar groups are presented in the left hand panel of Figure 4. Obviously the envelopes of the old disk stars with $M_{\mathbf{V}}$ $\geq+2$ have not experienced much convective mixing, while those of higher luminosity have been processed to a much greater extent. In this domain of the HR diagram it often is difficult to separate first ascent giants from clump giants. To illustrate this dilemma for the old disk stars we have plotted representations of the red giant branch and clump for the old but metal-rich $([\mathrm{Fe} / \mathrm{H}] \approx$ 0) open cluster M67 (Eggen and Sandage 1964), and the more metal-poor $([\mathrm{Fe} / \mathrm{H}] \approx-0.8)$ globular cluster 47 Tuc (Hesser et al. 1987). The old disk giants' HR diagram positions generally are contained between the branches of these clusters. This plot suggests that at least some of the more luminous old disk giants are helium core burning clump stars. It is impossible here to identify cleanly the clump stars, but pre- or post-core helium flash status does not seem to affect the isotope ratios of the old disk sample.

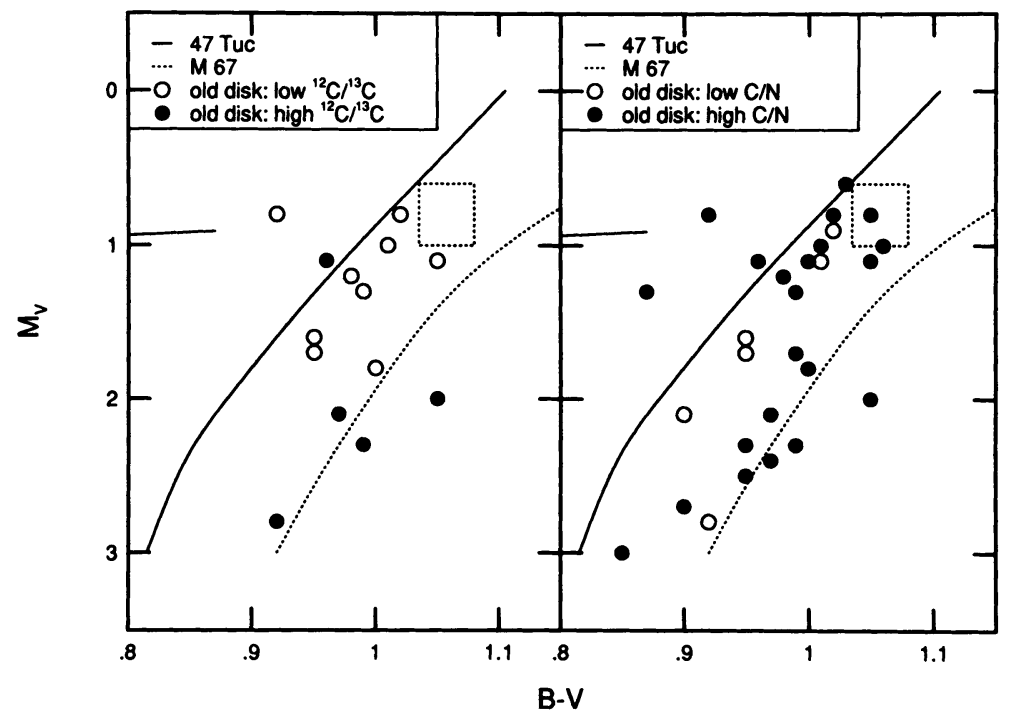

Figure 4. ${ }^{12} \mathrm{C} /{ }^{13} \mathrm{C}$ and $\mathrm{C} / \mathrm{N}$ ratios as functions of $\mathrm{HR}$ diagram positions for old disk stars. See the text for the descriptions of the divisions of the stars into high and low ratio groups in each panel. Positions of the giant branch and clump for M67 and the giant and horizontal branches for 47 Tuc have been indicated in both panels.

The $\mathrm{C} / \mathrm{N}$ ratios of these stars do not exhibit the same luminosity effect. In the right hand panel of Figure 4 we have plotted these ratios directly from the Cottrell and Sneden paper. The high $\mathrm{C} / \mathrm{N}$ 
ratios of these stars seem to be totally insensitive to giant branch position. It is hoped that new isotope ratio analyses of the full Cottrell-Sneden sample, along with determinations of $\mathbf{L i}$ abundances for these stars, will delineate more sharply the HR diagram location of the onset of convective mixing effects in old disk stars. Also, it would be quite helpful for someone to extend the old disk giant studies to cooler stars (Cottrell and Sneden limited their sample to G8-K1 giants) to study possible changes in the CNO element abundances near the giant branch tip of this stellar population.

For the globular cluster stars, we already have emphasized with the M13 data of Figure 2 that often the $\mathbf{C}$ depletions and especially the $\mathbf{N}$ enhancements along the giant branch are larger than those seen in young and old disk stars. Beyond this well known result, space limitations here force a focus only on consideration of a few recent papers that seem to yield significant new insights on the exploration of $\mathrm{C} / \mathrm{N}$ ratios. There have been a number of reviews of the complex behavior of $\mathrm{C}$ and $\mathrm{N}$ along the giant branches of globular clusters, highlighted by the excellent summary of Smith (1987). The reader is directed to his Table 2, which summarizes the general categories of $\mathrm{C}$ and $\mathrm{N}$ variations in clusters, and to the extensive discussion of hypotheses to explain these variations. These proposed explanations of course generally fall under the headings of: a) "internal mixing", for those clusters in which there seems to be a clear anticorrelation between the $\mathrm{C}$ and $\mathrm{N}$ abundances; and $b$ ) "primordial abundance variations", for those clusters with no obvious correlation between the $\mathrm{C}$ and $\mathrm{N}$ abundances (often taking the form of $\mathrm{N}$ abundances in some stars well in excess of those expected from the assumption of a constant initial total $\mathrm{C}+\mathrm{N}+\mathrm{O}$ ). At present neither process by itself seems to fit all the observed abundance variations, but the most recent work on globular clusters with moderate metal deficiencies $([\mathrm{Fe} / \mathrm{H}]>-1.5)$ suggests that both may have affected the CNO abundances of many evolved stars.

To begin, several studies have used low resolution spectroscopy for stars in the mildly metalpoor $([\mathrm{Fe} / \mathrm{H}] \approx-0.8)$ globular cluster 47 Tuc to show that star-to-star variations in the strengths of $\mathrm{CH}$ and $\mathrm{CN}$ bands occur throughout the giant and subgiant branches and even down to the main sequence turnoff; see for example Bell et al. (1983) and references therein. Clearly it is difficult to impose an explanation for these band strength variations based purely on internal mixing for stars so near the main sequence that they should not have developed convective envelopes of any consequence. New observations by Briley et al. (1990) of 47 Tuc main sequence stars demonstrates the persistence of the $\mathrm{CH}$ and $\mathrm{CN}$ variations to more than a magnitude below the turnoff. The $\mathrm{CN}$ band strengths apparently are distributed in the classical pattern of bi-modality that has been seen along the giant branches of other moderately metal-poor clusters. However, there seems to be no obvious anticorrelation between the $\mathrm{CH}$ and $\mathrm{CN}$ band strengths that would have been expected in the case of mixing of $\mathrm{CN}$-cycle processed material from the stellar interiors. At present the conclusion from this study seems inescapable: either $a$ ) the main sequence stars of 47 Tuc were formed from material that was inhomogeneous in $\mathrm{C}$ and $\mathrm{N}$ contents, or $b$ ) such material was transferred, by evolved mass losing stars that now are extinct, to the outer envelopes of the present main sequence stars after birth. This has been stated narrowly for 47 Tuc but presumably can apply equally well to other clusters with similar metallicities and evidences for $\mathrm{CN}$ bi-modality.

In apparent contrast to this result, Smith and Suntzeff (1990) have used moderate resolution CO spectra to determine carbon isotope ratios for a large number of giants of globular clusters M4 and NGC 6752, both of which show pronounced CN bi-modality. All giants of these clusters exhibit strong ${ }^{13} \mathrm{CO}$ bandheads, yielding very low isotope ratios: ${ }^{12} \mathrm{C} /{ }^{13} \mathrm{C}=4 \pm 1$, irrespective of $\mathrm{CN}$ band strengths. This result probably can be extended also to $47 \mathrm{Tuc}$, as Brown and Wallerstein (1989) and Bell et al. (1990) have shown that 5 giants of that cluster have ${ }^{12} \mathrm{C} /{ }^{13} \mathrm{C}<13$. The 
average ratio may be significantly less than 10 , but the ratios determined from the CO data of 47 Tuc (Bell et al. 1990) are quite sensitive to microturbulent velocity assumptions.

Smith and Suntzeff (1990) suggest that the resolution of all the $C / N$ and ${ }^{12} \mathrm{C} /{ }^{13} \mathrm{C}$ data in the $\mathrm{CN}$ bi-modal clusters may lie in a two-step process. First, winds from intermediate mass stars pollute the intracluster medium with varying amounts of $\mathrm{C}$ and $\mathrm{N}$, from which eventually low mass cluster dwarf stars with different $\mathrm{CH}$ and $\mathrm{CN}$ band strengths are created. Then during the long main sequence lifetimes of these low mass stars, slow circulation currents (of some sort) bring large fractions of their envelopes down to interior regions hot enough to process ${ }^{12} \mathrm{C}$ into ${ }^{13} \mathrm{C}$ via proton captures, but not necessarily deep enough to drain $\mathrm{C}$ into $\mathrm{N}$ (this partial $\mathrm{CN}$ cycle has of course previously been proposed for the old disk stars by other investigators). These steps finally create low mass giant stars with low carbon isotope ratios but widely varying $\mathrm{C} / \mathrm{N}$ ratios. Interestingly, Smith and Suntzeff (1990) also show that there is a tendency for decreasing total carbon abundances with increasing luminosities along the M4 and NGC 6752 giant branches. Note that their sample of giants essentially covers only the upper giant branch of each cluster: $-4 \leq M_{\mathrm{V}} \leq-1$. The first dredge up should be complete at lower luminosities than these values, and so once again it is apparent that some extra mixing event during the first ascent of the giant branch needs to be invoked to make sense of these results. It would be very helpful to know the ${ }^{12} \mathrm{C} /{ }^{13} \mathrm{C}$ ratios in lower luminosity stars in the globular clusters discussed here, but unfortunately the CO bandstrengths weaken considerably in the warmer, less luminous stars residing on the lower giant branches.

\section{Oxygen Abundances}

A discussion of the CNO group elements in giants would not be complete without some comments on the apparent star-to-star variations in [O/Fe] in stars of some globular clusters. For field metalpoor stars it has been understood for some time that $O$ is enhanced relative to $\mathrm{Fe}$ (e.g. Sneden et al. 1979), and the debate today centers only on whether the correct abundance ratio is a constant $[\mathrm{O} / \mathrm{Fe}] \approx+0.4$, (as deduced from giants: Gratton and Ortolani 1986, Barbuy 1988), or is as large as $[\mathrm{O} / \mathrm{Fe}] \approx+1$, possibly increasing with decreasing overall metallicity (as deduced from dwarfs: Abia and Rebolo 1989). For the globular clusters, Pilachowski et al. (1983) drew attention to possible cluster-to-cluster variations in [O/Fe], and more recent work has centered on star-to-star variations in this ratio within some clusters. For example, Pilachowski (1988) found a large $O$ deficiency in star VII-18 (relative to other stars of similar evolutionary state) in the very metal-poor cluster M92. She suggested that the sum of the abundances of the CNO group in the $6 \mathrm{M} 92$ giants of her sample is conserved, and that this fact argues for the mixing of both $\mathrm{CN}$ cycle and $\mathrm{ON}$ cycle products to the surfaces of these stars. Unfortunately, this idea probably cannot account for every giant that is deficient in O. Take the case of the star II-67 in M13: it apparently has an O abundance down by a factor of 5-10 relative to other cluster giants (Leep et al. 1986; Hatzes 1987). However, Langer et al. (1987) find that the $\mathrm{CN}, \mathrm{CH}$, and $\mathrm{NH}$ band strengths of this star are indistinguishable from those of M13 giants with normal $\mathrm{O}$ abundances, and thus probably no large amount of $\mathrm{ON}$ cycle material has reached the surface of this star.

Abundances of $\mathrm{Na}$ in the globular cluster giants complicate this picture considerably. Several studies previously had pointed out that there is a positive correlation between the strengths of $\mathrm{Na} I$ and $\mathrm{CN}$ features, and to some extent those of Al I also (see for example Cottrell and Da Costa 1981; Norris and Pilachowski 1985). This raises the small possibility that the physical structure of the atmosphere of metal-poor giants might not be well understood as yet. More importantly 
however this result suggests that efficient synthesis of $\mathrm{Na}$ from the reaction ${ }^{22} \mathrm{Ne}(\mathrm{p}, \gamma)^{23} \mathrm{Na}$ has occurred, but where is the synthesis site? Pilachowski (1988) and Paltoglou and Norris (1989) have pointed out that the $\mathrm{Na}$ abundances also seem to be anticorrelated with the $\mathrm{O}$ abundances in cluster giants. Denissenkov and Denissenkova (1990; see also their poster paper in this conference) claim that the Na synthesis can take place very rapidly in the interior regions in which the $\mathrm{ON}$ cycle has occurred. If there is mixing between the surface and the $\mathrm{ON}$ cycle layers then a natural consequence would be enhanced $\mathrm{Na}$ and depleted $\mathrm{O}$ in the red giant atmosphere. Unfortunately, the same effect could occur in the primordial abundance variation scheme, as one would merely need to postulate that the $\mathrm{O}$-poor red giants were seeded initially with material of such composition expelled from an intermediate mass star of the cluster that has now completed its evolution. Clearly abundance determinations of the CNONa elements in main sequence stars of globular clusters are needed to help understand whether primordial abundance variations or internal evolution is most important for the abundance mix of a globular cluster giant. A decent statistical base of such studies probably awaits the new generation of large optical reflectors.

\section{Conclusion}

In this brief review we have summarized the major trends of CNO abundances of stars undergoing the first ascent of the red giant branch. We have shown the differences in this abundance group in stars of different populations, emphasizing the fact that the departures of observed abundances from standard first dredge up theory appear to become larger as the stellar metallicities decrease. It seems apparent that some extra mixing event or events must be postulated to occur either during the main sequence lives of low mass stars or during the period in which they evolved along the giant branch. We are unable to identify that extra mixing event with precision at this time, but the observations seem refined enough now to warrant a fresh look at the light element synthesis in low mass stellar interiors.

\section{n. References}

Abia, C., and Rebolo, R. (1989) Astrophys. J., 347, 186.

Anders, E., and Grevesse, N. (1989) Geochim. Cosmochim. Acta, 53, 197.

Barbuy, B. (1988) Astron. Astrophys., 191, 121.

Bell, R. A., Briley, M. M., and Smith, G. H. (1990) Astron. J., 100, 187.

Bell, R. A., Hesser, J. E., and Cannon, R. D. (1983) Astrophys. J., 269, 580.

Boesgaard, A. M. (1989) Astrophys. J., 336, 798.

Briley, M. M., Hesser, J. E., and Bell, R. A. (1990) Astrophys. J., submitted.

Brown, J. A. (1987) Astrophys. J., 317, 701.

Brown, J. A., Sneden, C., Lambert, D. L., and Dutchover, E. Jr. (1989) Astrophys. J. Suppl., $71,293$.

Brown, J. A., and Wallerstein, G. (1989) Astron. J., 98, 1643.

Cayrel de Strobel, G., Bentolila, C., Hauck, B., and Dequennoy, A. (1984) Astron. Astrophys. Suppl., 59, 145.

Conti, P. S., Greenstein, J. L., Spinrad, H., Wallerstein, G., and Vardya, M. S. (1967) Astrophys. J., 148, 105.

Cottrell, P. S., and Da Costa, G. S. (1981) Astrophys. J. Lett., 245, L79. 
Cottrell, P. S., and Sneden, C. (1985) Astron. Astrophys., 161, 314.

Day, R. L., Lambert, D. L., and Sneden, C. (1973) Astrophys. J., 185, 213.

Dearborn, D. S. P. (1990) preprint.

Dearborn, D. S. P., Eggleton, P. P., and Schramm, D. N. (1976) Astrophys. J., 203, 455.

Dearborn, D. S. P., Lambert, D. L., and Tomkin, J. (1975) Astrophys. J., 200, 675 .

Denissenkov, P. A., and Denissenkova, S. N. (1990) Pis'ma Astron. Zh., in press.

Eggen, O. J., and Sandage, A. R. (1964) Astrophys. J., 140, 130.

Gilroy, K. K. (1989) Astrophys. J., 347, 835.

Gilroy, K. K., and Brown, J. A. (1990) preprint.

Gratton, R. G., and Ortolani, S. (1986) Astron. Astrophys., 169, 201.

Greene, T. F. (1969) Astrophys. J., 157, 737.

Harris, M. J., Lambert, D. L., and Smith, V. V. (1988) Astrophys. J., 325, 768.

Hatzes, A. P. (1987) Publ. Astron. Soc. Pacific, 99, 369.

Hesser, J. E., Harris, W. E., VandenBerg, D. A., Allwright, J. W. B., Shott, P., and Stetson, P. B. (1987) Publ. Astron. Soc. Pac., 99, 739.

Iben, I. Jr. (1967a) Astrophys. J., 147, 624.

Iben, I. Jr. (1967b) Astrophys. J., 147, 650.

Kjaergaard, P., Gustafsson, B., Walker, G. A. H., and Hultqvist, L. (1982) Astron. Astrophys., $115,145$.

Lambert, D. L. (1978) Mon. Not. Roy. Astron. Soc., 182, 249.

Lambert, D. L., and Dearborn, D. S. (1972) Mém. Soc. Roy. Sci. Liège, 6e Serie, 3, 147.

Lambert, D. L., and Ries, L. M. (1981) Astrophys. J., 248, 228.

Lambert, D. L., and Sneden, C. (1977) Astrophys. J., 215, 591.

Langer, G. E., Friel, E., Kraft, R. P., Suntzeff, N. B. (1987) Publ. Astron. Soc. Pacific, 99, 15.

Langer, G. E., Kraft, R. P., and Friel, E. (1985) Publ. Astron. Soc. Pacific, 97, 373.

Leep, E. M., Wallerstein, G., and Oke, J. B. (1986) Astron. J., 91, 1117.

Luck, R. E. (1990) Astrophys. J. Suppl., in press.

Norris, J., and Pilachowski, C. A. (1985) Astrophys. J., 299, 295.

Paltoglou, G., and Norris, J. E. (1989) Astrophys. J., 336, 185.

Pilachowski, C. A. (1988) in G. Cayrel de Strobel, M. Spite, and T. Lloyd Evans (eds.), The Abundance Spread Within Globular Clusters: Spectroscopy of Individual Stars, IAU General Assembly JCM 5 and CM 37/3, p. 1.

Pilachowski, C. A., Sneden, C., and Wallerstein, G. (1983) Astrophys. J. Suppl., 52, 241.

Smith, G. H. (1987) Publ. Astron. Soc. Pac., 99, 67.

Smith, V. V. (1990) in R. Pallavicini (ed.), High Resolution Spectroscopy in Astrophysics, Mem. Soc. Astr. Ital., in press.

Smith, V. V., and Suntzeff, N. B. (1989) Astron. J., 97, 1699.

Smith, V. V., and Suntzeff, N. B. (1990) preprint.

Sneden, C. (1973) Astrophys. J., 184, 839.

Sneden, C. (1974) Astrophys. J., 189, 493.

Sneden, C., Lambert, D. L., Tomkin, J., and Peterson, R. C. (1978) Astrophys. J., 222, 585.

Sneden, C., Lambert, D. L., and Whitaker, R. W. (1979) Astrophys. J., 234, 964.

Sneden, C., Pilachowski, C. A., and VandenBerg, D. A. (1986) Astrophys. J., 311, 826.

Suntzeff, N. B. (1981) Astrophys. J. Suppl., 47, 1.

Sweigart, A. V., and Mengel, J. G. (1979) Astrophys. J., 229, 624.

Tomkin, J., Lambert, D. L., and Luck, R. E. (1975) Astrophys. J., 199, 436.

Tomkin, J., Luck, R. E., and Lambert, D. L. (1976) Astrophys. J., 210, 694. 